ISSN: 2215-2644

\title{
Estrategias disposicionales y aprendizajes significativos en el aula virtual
}

\author{
Duarte- Herrera, Marcela; Montalvo Apolín, Danitza Elfi; Valdes Lozano, Dora Elia \\ Estrategias disposicionales y aprendizajes significativos en el aula virtual \\ Revista Educación, vol. 43, núm. 2, 2019 \\ Universidad de Costa Rica, Costa Rica \\ Disponible en: http://www.redalyc.org/articulo.oa?id=44058158038 \\ DOI: https://doi.org/10.15517/revedu.v43i2.34038
}

Esta obra está bajo una Licencia Creative Commons Atribución-NoComercial-SinDerivar 3.0 Internacional. 


\section{Estrategias disposicionales y aprendizajes significativos en el aula virtual}

Dispositional Strategies and Meaningful Learning in Virtual Classrooms

Marcela Duarte- Herrera

Universidad Santiago de Cali, Colombia

DOI: https://doi.org/10.15517/revedu.v43i2.34038

marcela.duarte00@usc.edu.co

(iD) http://orcid.org/0000-0001-5800-2381

Danitza Elfi Montalvo Apolin

Instituto Tecnológico y de Estudios Superiores de Monterrey,

México

danitza@tecvirtual.mx

(D) http://orcid.org/0000-0002-1087-4356

Dora Elia Valdes Lozano

Instituto Tecnológico y de Estudios Superiores de Monterrey,

México

dvaldes@itesm.mx

http://orcid.org/0000-0001-8343-4880

Recepción: 28 Enero 2019

Aprobación: 11 Junio 2019

\section{Resumen:}

Este estudio identificó las estrategias disposicionales empleadas por tutores en el diseño y la implementación de clases a nivel universitario, para promover aprendizajes significativos en un entorno virtual de aprendizaje en ocho diseños instruccionales analizados. Metodológicamente, se siguió el enfoque cualitativo-fenomenológico utilizando como instrumentos el análisis de contenido en cuatro aulas virtuales y entrevistas semiestructuradas efectuadas a una docente y a cinco estudiantes de Psicología en modalidad a distancia con apoyo del aula virtual de una institución universitaria. Se aplicaron cuatro tipos de matrices para el análisis de contenido, con las cuales se identificaron los aspectos motivacionales y los tipos de aprendizaje significativo que se presentan en los diseños instruccionales de los cursos analizados. Para validar el estudio se recurrió a un análisis de datos mediante el proceso de triangulación por instrumentos. Los resultados demostraron que (a) en algunos casos, se hace uso de estrategias disposicionales que promueven aprendizajes significativos con los contenidos discursivos de las actividades y de las retroalimentaciones mediante la comunicación sincrónica y asincrónica; (b) las estrategias que utiliza el cuerpo docente para motivar a sus estudiantes, no son el resultado de un diseño previo e intencionado sino de un proceso intuitivo; (c) las estrategias motivacionales que el profesorado emplea implican el uso de frases o imágenes que motivan a trabajar en los contenidos temáticos. Teniendo en cuenta los resultados del estudio, se recomienda para futuras investigaciones en el ámbito educativo sobre la modalidad a distancia, desarrollar e implementar procesos instruccionales previamente diseñados, donde el personal docente potencie tanto la comunicación efectiva como el proceso de aprendizaje, utilizando estrategias de enseñanza que promuevan la motivación al aprendizaje de modo intencional y pertinente para las dinámicas propias de las aulas virtuales, mostrando flexibilidad y adaptación a las necesidades del estudiantado.

Palabras Clave: Estrategias Educativas, Educación a Distancia, Enseñanza, Motivación, Práctica Educativa.

\section{Abstract:}

Dispositional strategies used by university instructors for class design and execution promotes meaningful learning in virtual classrooms. This study analyzes eight types of instructional designs used for university courses. A qualitative-phenomenological focus methodology was used in four virtual classrooms together with semi-structured interviews with one professor and five psychology students enrolled in a distance university program. Four types of matrixes were used for content analysis to identify motivational aspects and types of meaningful learning in the aforementioned instructional setting. Data analysis was used to validate this study through an instrument triangulation process. According to the results: (a) Dispositional strategies may promote meaningful learning with discursive content of the activities and feedback through synchronous and asynchronous 
communication. (b) Strategies used by the instructors to motivate students were not previously designed or intentional, but rather, the result of an intuitive process. (c) Motivational strategies used by the instructors use of phrases or images that motivate students to work on the thematic content. Based on the study results, it is recommended for purposes of future educational research on distance learning, to develop and execute previously designed instruction with effective communication fostered by the teaching staff to promote effective communication as well as learning and use teaching strategies that will motivate intentional learning pertinent for virtual classroom dynamics that demonstrates flexibility and adapts to student needs.

KEYWORDS: Educational Strategies, Distance Education, Teaching, Motivation, Educational Practices.

\section{INTRODUCCIÓN}

El profesorado de entornos virtuales de aprendizaje enfrenta retos y problemas ante las demandas, necesidades y particularidades del estudiantado que se forman a nivel universitario. Actualmente, ser docente en una plataforma virtual supone mediar y facilitar conocimiento, a través de diseños instruccionales y actividades que promuevan la construcción colectiva del conocimiento (Angulo, 2005; García, 2001; Maor, 2003). Este estudio surgió de las observaciones desarrolladas en un contexto educativo de educación a distancia de una institución colombiana, reconociendo las dificultades para establecer una relación de aprendizaje efectiva docente-estudiante a través de las tecnologías de la información y comunicación (TIC). Para ello, se tuvieron en cuenta los siguientes aspectos: (1) la necesidad de motivación del estudiantado como elemento relacionado con el desempeño académico; (2) la observación de las prácticas docentes en cuanto a su rol; (3) y las prácticas docentes dispares, especialmente en lo que refiere a los medios para promover motivación al aprendizaje.

\section{ANTECEDENTES PRÁCTICOS}

García, Loredo y Carranza (2008), proponen un modelo de evaluación y formación del personal docente en Educación Superior, a partir del seguimiento de la práctica educativa. Otros autores como Padilla, Rincón y Lagos (2016), se cuestionan sobre la coherencia de los modelos pedagógicos en la educación superior y el uso de las TIC, señalando la importancia del cuerpo docente como medio para brindar herramientas conceptuales, tecnológicas y operativas al estudiantado. En cuanto a las estrategias discursivas de interacción, se encuentran en el monitoreo del maestro hacia el estudiantado para facilitar el discurso, verificar la comprensión, motivar, participar y resolver dudas (Cañedo y Figueroa, 2013). El aprendizaje significativo se fomenta y debe afianzarse a través de actividades independientes y en equipo. Pegalajar (2016), menciona que el estudiantado universitario dispone de "...una adecuada capacidad para tomar decisiones, planificar, autoevaluar el propio desempeño y autorregular sus actuaciones” (p. 12).

Referente a las estrategias disposicionales de aprendizaje, Anaya y Anaya (2010), retoman las necesidades y motivaciones propias, destacando que una cierta dosis de estrés, puede ser de utilidad siempre que cada docente pueda potenciar las necesidades de seguridad, autoaprendizaje, desarrollo y trascendencia. Valenzuela y Pérez (2013), destacan cómo los entornos virtuales permiten establecer seguimiento y control del desempeño del estudiantado, haciendo énfasis en la barrera que representa la falta de contacto directo entre maestro y aprendiz.

Herrera (2014) encontró que la motivación por el estudio está dada por intereses personales y creencias de control. Carvalho y De Oliveira (2015), señalan que el uso de aulas virtuales requiere de un/a aprendiz/a autodeterminado/a, siendo el profesorado mediador del aprendizaje. Hellberg y Lindberg (2017), destacan cómo el aspecto motivacional intrínseco está mediado por la etapa del ciclo vital en la cual se encuentre el estudiantado, así como la forma en la cual se presenten las actividades. Moreira (2000) refiere que el profesorado es un mediador entre los saberes previos del estudiantado y la organización o sentido que da al nuevo material o concepto a trabajar. Arias y Oblitas (2014) destacan el desempeño del alumnado con 
el modelo de aprendizaje significativo obtienen mejores desempeños en la educación superior, cuestión que también debe considerarse para la orientación de cada docente en entornos virtuales. Ahmed, Ahmed, Miller, Gebremeskel y Ebessa (2018) reconocieron en su estudio el valor de las actividades de aprendizaje significativo, en población que ha tenido problemas de acceso, permanencia y calidad en el sistema educativo. De igual forma Martins y Unger (2017), destacan el uso de la tecnología para disminuir las dificultades de acceso a la educación, requiriendo del interés y formación pedagógica del maestro para potenciar estos espacios.

\section{REFERENTES CONCEPTUALES}

La educación a distancia es un sistema tecnológico de comunicación en múltiples vías, donde se despliegan los recursos didácticos y de apoyo, según la acción propuesta por el personal docente encargado del curso (García, 2001). Esto implicaría que docente y estudiante tendrían que establecer regularidades en el proceso de comunicación, sin requerir el continuo encuentro presencial, lo cual ha impulsado esta modalidad educativa, como medio para que más personas puedan acceder a procesos formales en la educación básica, media y superior (Hwang, 2017).

En años anteriores Angulo (2005) señalaba que, si bien es una modalidad y estrategia de aprendizaje accesible e incluyente, aún presenta fallas en su implementación mientras no existan políticas claras al respecto, así como una formación intencionada de las y los docentes en su manejo. Estas ideas persisten actualmente cuando se revisan estudios de los últimos años que demuestran que hay interés de los entes educativos por usar sesiones de aprendizaje virtuales, pero existe ciertos desconocimientos de la efectividad de las plataformas y su uso continuado, de modo que esto podría evitar confirmar parcialmente lo trascendente de la modalidad virtual en la transmisión del conocimiento y en la adquisición del aprendizaje significativo. Por ejemplo, en el estudio de Marín y Maldonado (2011), el estudiantado de educación considera las plataformas virtuales como un medio para el aprendizaje significativo y colaborativo, facilitando el trabajo flexible, adaptado a sus necesidades. Por otro lado, García (2017) destaca el lugar que ha adquirido en la educación formal el uso de las plataformas virtuales, donde las efectividades de las estrategias empleadas por el profesorado son igual de efectivas que en la educación presencial. El fácil acceso y la flexibilidad horaria, adaptable a las necesidades individuales, resultan factores atractivos para el estudiantado, pero ¿el cuerpo docente está preparado para adaptar sus propuestas pedagógicas con estrategias que motiven el interés del estudiantado?

Las estrategias de aprendizaje son una serie de acciones intencionadas, cuyo objeto final es facilitar ambientes de aprendizajes determinados y significativos, posibilitar el autocontrol del aprendiz y cualificar el quehacer docente (Monereo, 2000; Vivas, 2010). Para ello, el instructor o docente, tendría que considerar las características de los aprendices, de acuerdo con sus capacidades y habilidades cognitivas, los hábitos de trabajo intelectual, técnicas y métodos de estudio y procedimientos para resolver problemas (Díaz y Hernández, 2002).

Las estrategias disposicionales son aquellas que permiten mantener el esfuerzo, el interés y/o la motivación del estudiantado a lo largo del proceso de ejecución de una tarea, estas se dividen en dos grupos según Monereo (2000), Estrategias afectivas y de automanejo, y Estrategias de control de contexto. En este estudio se hizo énfasis en la primera categoría la cual se refiere a los procesos motivacionales y afectivos que median el aprendizaje. Es importante que por medio de estas estrategias se fomente la regulación de emociones inmersas en el aprendizaje (ansiedad) y la autoconfianza en la capacidad de ejecutar la tarea del aprendiz (Tintaya, 2010). Gargallo (2000) hizo énfasis en la conexión entre motivación y afecto como uno de los niveles de procesamiento y control cognitivo exigido en el proceso de enseñanza-aprendizaje. Por ello, la creación de un ambiente que disminuya los niveles de ansiedad, limite las distracciones y permita delimitar prioridades 
de aprendizaje significativo deben ser factores cognitivos y emocionales prioritarios en el aula tanto para el grupo docente como para el estudiantado.

El aprendizaje significativo es aquel que logra la conexión cognitiva y emocional entre los contenidos impartidos por el profesorado y la intención de aprendizaje y experiencia previa de cada aprendiz (Díaz y Hernández, 2002), y el o la docente que trabaja con estos preceptos, reconoce las características de su estudiante y facilita dichas conexiones. Los clásicos de las teorías de aprendizaje como Ausubel, Novak y Hanesian (1983) propusieron tres tipos de aprendizaje significativo, según el tipo de contenido o ámbito de incidencia: (1) El aprendizaje de representaciones que hace referencia al tipo más simple de aprendizaje significativo, cuando el aprendiz asocia un símbolo arbitrario con un objeto, situación o concepto que le resulta similar o familiar, (2) El aprendizaje de conceptos que alude a la formación o asimilación de redes representacionales que tienen criterios de definición comunes, y por tanto, se les asigna un mismo símbolo o signo y (3) El aprendizaje de proposiciones, que implica que el aprendiz establece redes de relación entre varios conceptos que producen un significado que, en conjunto, es asimilado por la estructura cognoscitiva.

\section{Procedimientos metodológicos}

Se optó por un estudio cualitativo fenomenológico al tener como foco de interés la realidad humana, los eventos concretos e ideales tal como se presentan, sin condicionamientos de la teoría (Mieles, Tonon y Alvarado, 2012), y los entornos naturales del aprendizaje virtual para indagar las vivencias y los puntos de vista de las personas participantes, asunto que permitió aproximarse a la comprensión del fenómeno educativo como es el diseño de estrategias docentes y las experiencias del aula entre docentes y estudiantes. Se utilizó el análisis de contenido, con cuatro matrices distintas que se enfocaron a obtener información relativa a la motivación y al aprendizaje significativo. El muestreo por conveniencia, permitió convocar quienes cumplen los criterios requeridos para la investigación, accediendo libremente a facilitar la información requerida y a través de su firma de consentimiento informado (Giroux y Tremblay, 2009; Hernández, Fernández y Baptista, 2010).

De acuerdo con autores como Andreú (2001), Bardin (2002) e Íñiguez (2006), a través de la técnica del análisis de contenido, es posible interpretar material o producción textual, información simbólica (imágenes) o mensajes inmersos en el contexto, estableciendo una serie de categorías o reglas de codificación, que permitan identificar los aspectos relevantes para el objetivo de estudio; características que se trabajaron con el fin de inferir las estrategias disposicionales diseñadas e implementadas por el cuerpo docente, en el material instruccional presentado en las ocho unidades de aprendizaje de sus cursos.

Se seleccionó la entrevista semiestructurada de acuerdo con la opinión de Hernández et ál. (2010), conceptualizando este tipo de entrevista como una técnica flexible para obtener la información necesaria y responder a los objetivos del estudio, al mismo tiempo permitió ampliar o redireccionar el discurso de las personas entrevistadas.

\section{ANÁLISIS Y DISCUSIÓN DE RESULTADOS}

A continuación, se presentan los resultados obtenidos mediante el análisis de los elementos instruccionales y discursivos de la plataforma tecnológica utilizada por la docente y sus estudiantes, en torno de las estrategias disposicionales, el aprendizaje significativo y la relación que se establece entre el tutor y el aprendiz en un ambiente virtual. 
TABLA 1

Resultados del análisis de contenido de las aulas virtuales y estrategias disposicionales

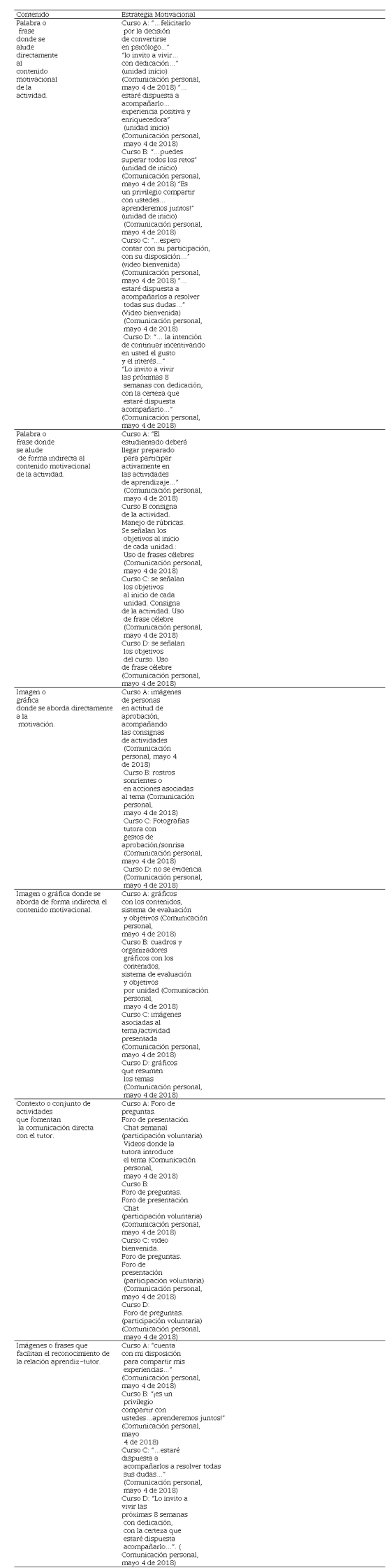




\section{Fuente: Elaboración propia}

En los textos e imágenes analizados, se observó que las actividades de bienvenida aluden a la motivación de tipo afectivo, especialmente en las unidades de inicio de curso. Como elemento común de comunicación y evidencia de la relación tutor-aprendiz, se encontraron las interacciones en los foros de preguntas como forma de establecer comunicación sincrónica y asincrónica en todos los cursos. Por otro lado, en las prácticas de enseñanza la motivación del grupo docente, se favoreció en el discurso el mantenimiento de la presencia afectiva y ayudó a mantener el esfuerzo del estudiantado.

Como se puede notar en la Tabla 1, las estrategias disposicionales que aluden a la motivación extrínseca, son comunes. El personal docente presenta los objetivos, las consignas, los organizadores gráficos y los dibujos alusivos al tema como recursos cognitivos de apoyo en las tareas. Se hace uso recurrente de frases sugestivas para evidenciar la importancia de la participación del estudiantado.

En la Tabla 2, se muestra la motivación y las emociones inmersas en relación tutor-aprendiz, y como ello alude al aprendizaje significativo que promueven las estrategias disposicionales de aprendizaje (Arias y Oblitas, 2014; Ahmed et ál., 2018; Díaz y Hernández, 2002; Hellberg y Linderberg, 2017; Maor, 2003; y Monereo, 2000).

TABLA 2

Resultados de análisis de contenido aulas virtuales - Aprendizaje Significativo

\begin{tabular}{|c|c|c|c|}
\hline $\begin{array}{l}\text { Tipo de Aprendizaje Significativo } \\
\text { en la } \\
\text { Estrategia de Aprendizaje Disposicionales }\end{array}$ & Sección / Unidad de Aprendizaje & Actividades & $\begin{array}{l}\text { Otros } \\
\text { elementos } \\
\text { del curso }\end{array}$ \\
\hline $\begin{array}{l}\text { Tipo 1. Aprendizaje } \\
\text { de representaciones. }\end{array}$ & $\begin{array}{l}\text { Curso A: } \\
\text { No se evidencian } \\
\text { Curso B: Introducción. Comprensión y construcción. } \\
\text { Reflexión } \\
\text { Curso C: Distintas secciones } \\
\text { por unidad } \\
\text { Curso D: Unidad de inicio }\end{array}$ & $\begin{array}{l}\text { Curso A: } \\
\text { No se evidencian. } \\
\text { Curso B: } \\
\text { No se evidencian. } \\
\text { Curso C: } \\
\text { No se evidencian. } \\
\text { Curso D: } \\
\text { No se evidencian }\end{array}$ & $\begin{array}{l}\text { Curso A: } \\
\text { No se evidencian } \\
\text { Curso B: } \\
\text { Dibujos asociados al tema } \\
\text { Curso C: } \\
\text { Dibujos asociados al tema. } \\
\text { Curso D: } \\
\text { Dibujos o gráficas asociadas al tema }\end{array}$ \\
\hline $\begin{array}{l}\text { Tipo 2. Formación } \\
\text { de } \\
\text { Conceptos. }\end{array}$ & $\begin{array}{l}\text { Curso A: Introducción al tema. Trabajo entrega } \\
\text { semanal } \\
\text { Curso B: Introducción } \\
\text { Curso C: Conociendo. Reflexionando. Mis fortalezas. } \\
\text { Curso D: Actividades }\end{array}$ & $\begin{array}{l}\text { Curso A: Video-exposición } \\
\text { del tema (estudiante). Quiz presencial. } \\
\text { Curso B: Preguntas asociadas a experiencia personal } \\
\text { para introducir } \\
\text { el tema. Examen Virtual } \\
\text { Curso C: Preguntas generadoras. Quiz virtual. } \\
\text { Curso } D \text { : } \\
\text { se señala } \\
\text { quizen } \\
\text { tutoria presencial }\end{array}$ & $\begin{array}{l}\text { Curso A: } \\
\text { Videos } \\
\text { tutoriales } \\
\text { del tema (hechos por la tutora). } \\
\text { Curso B: } \\
\text { videos } \\
\text { tuturiales } \\
\text { Curso C: } \\
\text { video : } \\
\text { alusivo } \\
\text { al tema } \\
\text { Curso D: } \\
\text { no se } \\
\text { evidencian }\end{array}$ \\
\hline $\begin{array}{l}\text { Tipo 3. Aprendizaje } \\
\text { de Proposiciones. }\end{array}$ & $\begin{array}{l}\text { Curso A: Trabajo entrega semanal. } \\
\text { Curso B: Aplicación } \\
\text { Curso C: Mis fortalezas. Aplicando } \\
\text { Curso D: Paralelo. Trabajo colaborativo }\end{array}$ & $\begin{array}{l}\text { Curso A: } \\
\text { Foro de socialización del tema -Mapa conceptual. } \\
\text { Ensayo Trabajo prueba } \\
\text { mini-mental (colaborativo) } \\
\text { Curso B: Trabajo de Campo (colaborativo e } \\
\text { individual) } \\
\text { Curso C: Mapas conceptuales. Foro para } \\
\text { construcción proyecto (colaborativo) } \\
\text { Curso D: Cuadro comparativo. Trabajo colaborativo } \\
\text { (foro) }\end{array}$ & $\begin{array}{l}\text { Curso A: } \\
\text { tutora retroalimenta } \\
\text { de acuerdo } \\
\text { con rúbrica } \\
\text { Curso B: } \\
\text { tutora retroalimenta } \\
\text { de acuerdo } \\
\text { con rúbrica } \\
\text { Curso C: retroalimentación } \\
\text { Curso D: retroalimentación según } \\
\text { rúbrica }\end{array}$ \\
\hline
\end{tabular}

Fuente: Elaboración propia

Se encuentra una tendencia a presentar actividades y elementos de apoyo en las unidades que promueven aprendizajes significativos, tales como el uso de retroalimentaciones en los procesos de evaluación. Dentro de este tipo de actividades, destaca la presencia de los trabajos colaborativos y los organizadores mentales (mapas conceptuales y cuadros comparativos).

El uso de imágenes alusivas al tema es el único elemento que alude a los aprendizajes significativos tipo uno en todos los diseños instruccionales. Dentro de las actividades de aprendizaje significativo tipo dos, se encuentra el uso del Quiz, donde en dos de los cursos se señala su implementación en la tutoría presencial. El uso de videos para mostrar conceptos, se observa en tres de los cursos, cuestión que también apelaría a aprendizajes significativos. 
Teniendo en cuenta lo aportado por Ausubel et ál. (1983) en lo que refiere a espacios de promoción de aprendizajes significativos, se detectó que las estrategias de aprendizaje disposicionales, se centran en actividades y elementos de apoyo para la formación de conceptos y el aprendizaje de proposiciones. Cada docente juega el papel de mediador del proceso enseñanza-aprendizaje, destacando el uso de la retroalimentación de las actividades como elemento de apoyo y el uso de videos (en tres casos) como medio de conectar al estudiante con el componente cognitivo, afectivo y experiencial de la actividad (Arias y Oblitas, 2014; Maor, 2003; Díaz y Hernández, 2002; y Meza, Torres y Lara, 2016).

Con la finalidad de reconocer los puntos de vista de tutores y aprendices sobre el proceso de diseño e implementación de estrategias disposicionales que promuevan el aprendizaje significativo en el aula virtual, se efectuó una entrevista a una docente del programa de psicología de la sede, y de acuerdo con los mismos ejes temáticos, se indagó la percepción de cinco estudiantes (ver Tabla 3 y Tabla 4), de modo que pudiesen contrastarse estos puntos de vistas con lo encontrado en los diseños instruccionales.

TABLA 3

Resultados de la entrevista semiestructurada a la docente

\begin{tabular}{|c|c|}
\hline Ejes temáticos & Respuestas \\
\hline $\begin{array}{l}\text { Proceso de diseño de } \\
\text { estrategias } \\
\text { disposicionales de } \\
\text { aprendizaje en el } \\
\text { Aula Virtual. }\end{array}$ & $\begin{array}{l}\text { Docente A: "... al momento de diseñar las actividades...en } \\
\text { primer momento lo que buscamos plantear es un objetivo de } \\
\text { la actividad y lograr que el estudiantado se sienta atraído por } \\
\text { el tema. Lamentablemente, el tiempo para el diseño de estas } \\
\text { actividades no es el que corresponde para toda su } \\
\text { planificación y todo su desarrollo...pero lo que se busca } \\
\text { primero es, fomentar el trabajo autoformativo" } \\
\text { (Comunicación personal, mayo } 15 \text { de } 2018 \text { ) "... tenemos el } \\
\text { foro para interactuar, se diseña para que el estudiantado se } \\
\text { plantee preguntas sobre el tema, para que pueda compartir } \\
\text { con sus compañeros...y la idea es motivarlos" "...la } \\
\text { evaluación para el curso en mi caso, es una evaluación } \\
\text { continua, que tiene un componente fundamental, la } \\
\text { retroalimentación" (Comunicación personal, mayo } 15 \text { de } \\
\text { 2018) }\end{array}$ \\
\hline $\begin{array}{l}\text { Manejo de la } \\
\text { Motivación en el Aula } \\
\text { Virtual. }\end{array}$ & $\begin{array}{l}\text { Docente A: "el elemento clave es la interacción, de hecho es la } \\
\text { oportunidad que tiene un docente de no tener un simple } \\
\text { papel de transmisión...el aula virtual nos permite una } \\
\text { interacción constante, y por tanto es un modelo } \\
\text { constructivista, permite una construcción del conocimiento" } \\
\text { (Comunicación personal, mayo } 15 \text { de 2018) }\end{array}$ \\
\hline $\begin{array}{l}\text { Relación Tutor- } \\
\text { Aprendiz en el Aula } \\
\text { Virtual. }\end{array}$ & $\begin{array}{l}\text { Docente A: "Cuando la interacción está ausente, el aula virtual } \\
\text { no tiene sentido, se convierte en un espacio donde se monta } \\
\text { material" "nuestra responsabilidad es reconocer la necesidad } \\
\text { del estudiantado" (Comunicación personal, mayo } 15 \text { de } \\
\text { 2018) }\end{array}$ \\
\hline $\begin{array}{l}\text { Proceso de } \\
\text { implementación de } \\
\text { estrategias } \\
\text { disposicionales de } \\
\text { aprendizaje en el } \\
\text { Aula Virtual. }\end{array}$ & $\begin{array}{l}\text { Docente A: "al estudiante se le pide llevar ese tema a su } \\
\text { contexto real" "hay un diseño, una planificación... pero en } \\
\text { realidad ese diseño puede estar muy claro... pero si no tiene el } \\
\text { componente de interacción no tiene sentido" (Comunicación } \\
\text { personal, mayo } 15 \text { de 2018) }\end{array}$ \\
\hline $\begin{array}{l}\text { Tipos/Medios que } \\
\text { promueven el } \\
\text { Aprendizaje } \\
\text { Significativo- } \\
\text { Relación con } \\
\text { Estrategias } \\
\text { disposicionales de } \\
\text { aprendizaje. }\end{array}$ & $\begin{array}{l}\text { Docente A: "la plataforma virtual en su totalidad creo que } \\
\text { promueve el aprendizaje significativo, pero haciendo uso de } \\
\text { todas las herramientas que tiene la plataforma... lo que } \\
\text { sucede es que las y los docentes también requieren unos } \\
\text { procesos formativos para trabajar en la virtualidad" "muchos } \\
\text { tutores no hacen uso del aula virtual, se quedan solo con el } \\
\text { espacio de la presencialidad" (Comunicación personal, mayo } \\
15 \text { de 2018) }\end{array}$ \\
\hline
\end{tabular}


En el diálogo con la maestra, ella comentó sobre el valor del ejercicio de planeación de actividades, comunicación y retroalimentación al estudiante en los procedimientos que se desarrollan en el aula virtual. Tanto en el proceso de diseño como de implementación de estrategias disposicionales de aprendizaje se muestra la importancia de establecer una comunicación clara y constante, que permite la contextualización del aprendizaje y permite que el aprendiz se sienta atraído e interesado en el contenido temático (destaca el chat como herramienta útil para aclarar dudas y promover la motivación). Según declara, esto es posible con las herramientas proporcionadas por la plataforma de aprendizaje, pero se requiere que el cuerpo docente conozca estas y haga uso de ellas de forma efectiva para generar aprendizajes significativos.

En el proceso de diseño, la docente presentó un acercamiento hacia los planteamientos de García et ál. (2008) y de Cañedo y Figueroa (2013) sobre la importancia de la planeación de la práctica educativa, la aplicación de las estrategias discursivas en el aula y la formación y seguimiento continuo del y la docente. En la implementación de estrategias se detectó la preocupación por la comunicación constante y la retroalimentación con apoyo de herramientas virtuales, aun cuando estos son asuntos comunes en la educación no se pueden minimizar, puesto que, solo de esta forma es posible conocer al estudiante y establecer una relación de co-construcción propia del aprendizaje significativo (Meza et ál., 2016, Valenzuela y Pérez, 2013).

La docente manifestó, además, que el entorno virtual puede promover el aprendizaje significativo si se hace uso de todas sus herramientas, como Wikis, Blogs, juegos en línea, entre otras. Esto podría resultar ser el medio más adecuado para poner al contexto lo aprendido (Arias y Oblitas, 2014), cuestión que podría relacionarse con los resultados del estudio de Landa, Silva y Guarnero (2012), donde podían observarse mejores desempeños en estudiantes de modalidad virtual versus estudiantes de modalidad presencial. Sin embargo, la entrevistada señaló que es importante el proceso formativo y de capacitación del personal docente para el adecuado aprovechamiento de las plataformas virtuales. 
Revista Educación, 2019, 43(2), July-December, ISSN: 0379-7082 / 2215-2644

TABLA 4

Resultados de las entrevistas semiestructurada a estudiantes

\begin{tabular}{|c|c|}
\hline Ejes temáticos & Respuestas \\
\hline $\begin{array}{l}\text { Proceso de } \\
\text { diseño de } \\
\text { estrategias } \\
\text { disposicionales } \\
\text { de aprendizaje } \\
\text { en el Aula } \\
\text { Virtual. }\end{array}$ & 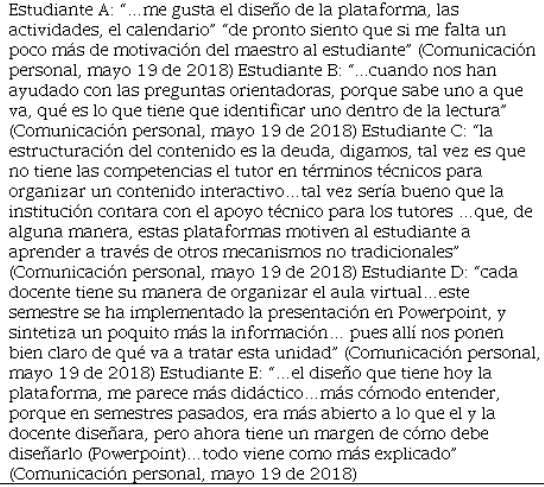 \\
\hline $\begin{array}{l}\text { Manejo de la } \\
\text { Motivación en el } \\
\text { Aula Virtual. }\end{array}$ & 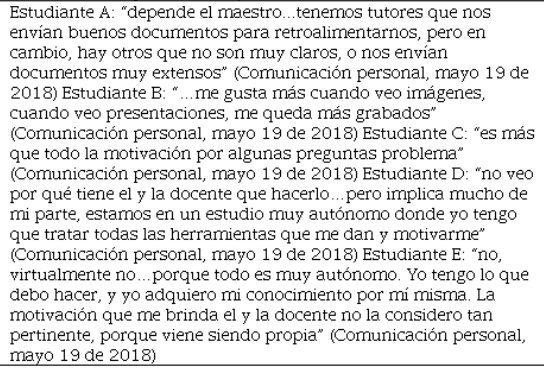 \\
\hline $\begin{array}{l}\text { Relación Tutor- } \\
\text { Aprendiz en el } \\
\text { Aula Virtual. }\end{array}$ & 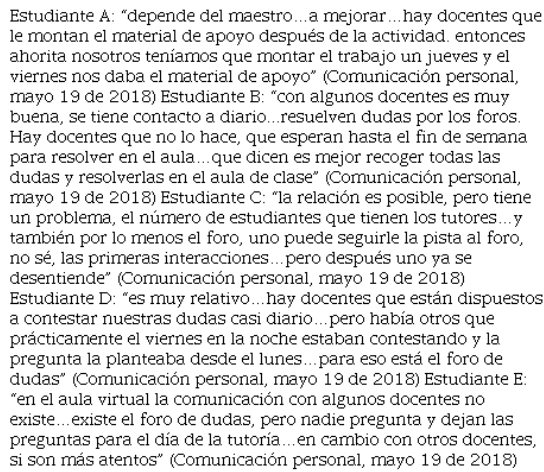 \\
\hline $\begin{array}{l}\text { Proceso de } \\
\text { implementación } \\
\text { de estrategias } \\
\text { disposicionales } \\
\text { de aprendizaje } \\
\text { en el Aula } \\
\text { Virtual. }\end{array}$ & 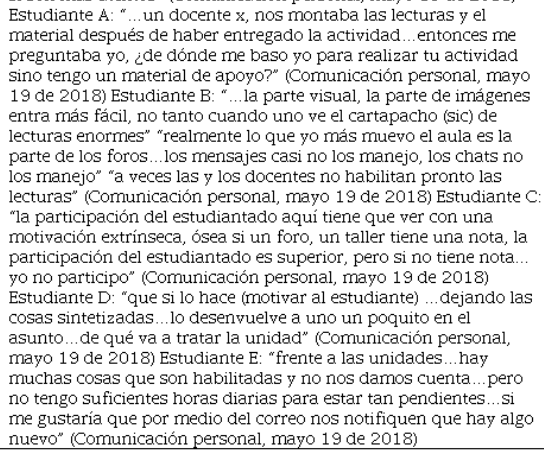 \\
\hline $\begin{array}{l}\text { Tipos/Medios } \\
\text { que promueven } \\
\text { el Aprendizaje } \\
\text { Significativo - } \\
\text { Relación con } \\
\text { Estrategias } \\
\text { disposicionales } \\
\text { de aprendizaje. }\end{array}$ & 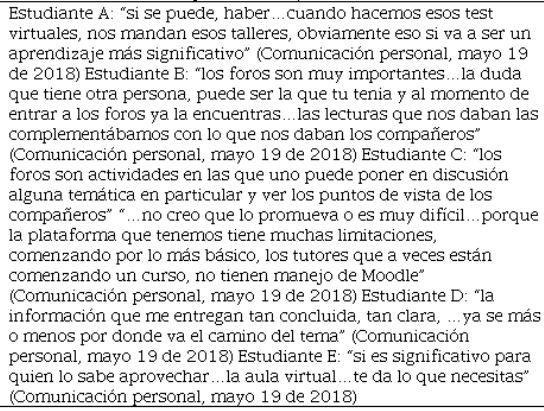 \\
\hline
\end{tabular}


El estudiantado entrevistado comentó que existen variaciones frente al diseño y al uso de estrategias disposicionales del aprendizaje que promuevan aprendizajes significativos en el entorno virtual, entre el colectivo docente del programa. En cuatro de los casos, estudiantes manifestaron que en la relación tutoraprendiz se da de manifestó que los foros son de utilidad para la comunicación pero que el número de estudiantes y las respectivas interacciones que se presentan en el foro hacen compleja la comunicación.

Referente al diseño de estrategias disposicionales se encuentran distintos elementos que el alumnado identificó como promotores de la motivación, como el diseño de las aulas, las preguntas orientadoras, así como la puesta del diseño instruccional en una presentación en formato digital. Uno de los entrevistados señaló que la estructuración del contenido debería ser responsabilidad de la universidad y no de los tutores, dada las limitadas competencias técnicas de estos. Frente al proceso de implementación de dichas estrategias, en cuatro de los casos, se destacó la claridad y oportunidad en que el tutor despliega la información, así como los medios por los cuales el estudiantado puede saber oportunamente que las actividades se encuentran habilitadas en la plataforma.

En relación con el aprendizaje significativo y las estrategias disposicionales, los participantes identificaron las actividades que se plantean en la plataforma como facilitadoras de este tipo de aprendizaje, destacando en dos de los casos, el uso de foros. En una de las entrevistas se señaló la dificultad de hacer uso adecuado de estas herramientas por la poca capacitación técnica de los tutores en el uso de las herramientas tecnológicas. En todos los casos, se destacó la importancia de la claridad en la información suministrada por parte del y la docente.

En cuanto al proceso de diseño e implementación de estas estrategias, las y los estudiantes mencionaron que existe variación de tutor a tutor, señalando los distintos tipos de diseño y regularidad en la comunicación, siendo este último, factor fundamental en el rol del y la docente como mediador de la motivación (Cañedo y Figueroa, 2013; Díaz y Hernández, 2002 y Meza et ál., 2016). Uno de los entrevistados enfatizó en la falta de capacitación de los tutores en el manejo de plataforma, cuestión que se considera fundamental para el desarrollo de la práctica educativa (García et ál., 2008; Valenzuela y Pérez, 2013) y cuestión, por la cual argumentó, se limita el aprendizaje significativo.

La motivación fue asociada por los entrevistados como un factor propio del estudiantado, asunto que se relaciona con los estudios de Anaya et ál. (2010), Boza y Toscano (2012) y Herrera (2014). Sin embargo, señalaron a los foros como medios de actividades que permiten la interacción (efectiva o no) con el tutor; dos de ellos, los asociaron con la posibilidad de obtener un aprendizaje significativo de acuerdo con el valor de la interacción tutor-aprendiz (Meza et ál., 2016).

En relación con la identificación del proceso de diseño de estrategias disposicionales, las planeaciones de estas en los contenidos instruccionales analizados no se muestran explícitas, tampoco en las percepciones de la docente y el estudiantado entrevistado. El reconocimiento de la implementación de dichas estrategias se ve reflejado en los contenidos instruccionales, así como en las entrevistas ejecutadas, aunque no explícitamente planeadas, identificando su uso como un factor que facilita el aprendizaje significativo del estudiantado. Díaz y Hernández (2002) y Monereo (2000) y Herrera (2014) demostraron que el uso de las estrategias fomenta la mantención del esfuerzo de parte del estudiantado, cuestión que puede asociarse con mejores desempeños académicos. En esta medida, se considera necesario generar estrategias de formación y acompañamiento al docente para optimizar el proceso de la instrucción, la motivación y el aprendizaje significativo (Cañedo y Figueroa, 2013; García et ál., 2008).

Se triangularon los datos recabados mediante un análisis de categorías, subcategorías e instrumentos de investigación (dos rejillas de análisis del contenido y entrevistas semiestructuradas a una docente y cinco estudiantes) de acuerdo con los planteamientos de Aguilar y Barroso (2015), así como los de Okuda y Gómez (2005). La Tabla 5 muestra los patrones de convergencia y divergencia de la información recabada. 
TABLA 5

Triangulación de datos

\begin{tabular}{|c|c|c|c|c|}
\hline $\begin{array}{l}\text { Instrumentos Categorías } \\
\text { de Análisis }\end{array}$ & $\begin{array}{l}\text { Matriz de } \\
\text { Estrategias } \\
\text { Disposicionales }\end{array}$ & $\begin{array}{l}\text { Matriz de } \\
\text { Aprendizaje } \\
\text { Significativo }\end{array}$ & $\begin{array}{l}\text { Entrevista } \\
\text { Tutora }\end{array}$ & $\begin{array}{l}\text { Entrevista } \\
\text { Estudiantes }\end{array}$ \\
\hline $\begin{array}{l}\text { Diseño estrategias } \\
\text { disposicionales }\end{array}$ & $\begin{array}{l}\text { Unidad de inicio: } \\
\text { Actividades de } \\
\text { Curso }\end{array}$ & $\begin{array}{l}\text { Aprendizaje } \\
\text { significativo }\end{array}$ & $\begin{array}{l}\text { Diseño de } \\
\text { actividades } \\
\text { que } \\
\text { despierten el } \\
\text { interés del } \\
\text { estudiantado }\end{array}$ & $\begin{array}{l}\text { Asociada a la } \\
\text { estructuración del } \\
\text { contenido por } \\
\text { parte del y la } \\
\text { docente }\end{array}$ \\
\hline $\begin{array}{l}\text { Implementación de } \\
\text { estrategias disposicionales }\end{array}$ & $\begin{array}{l}\text { Actividades de } \\
\text { interacción y } \\
\text { retroalimentación } \\
\text { de actividades }\end{array}$ & $\begin{array}{l}\text { Aprendizaje } \\
\text { significativo. } \\
\text { Retroalimentación } \\
\text { del tutor }\end{array}$ & $\begin{array}{l}\text { Poner en } \\
\text { contexto lo } \\
\text { aprendido } \\
\text { (actividades). }\end{array}$ & $\begin{array}{l}\text { Actividades } \\
\text { accesible entrega } \\
\text { de material } \\
\text { oportuna (tutor)- } \\
\text { foros }\end{array}$ \\
\hline Relación Tutor-Aprendiz & $\begin{array}{l}\text { Propuesta por el } \\
\text { tutor - } \\
\text { interacción } \\
\text { opcional- foros, } \\
\text { chat, videos }\end{array}$ & $\begin{array}{l}\text { Aprendizaje } \\
\text { significativo } \\
\text { Retroalimentación } \\
\text { del tutor }\end{array}$ & $\begin{array}{l}\text { Sincrónica y } \\
\text { asincrónica - } \\
\text { propuesta } \\
\text { por el tutor - } \\
\text { foros y chat } \\
\text { - conocer al } \\
\text { estudiante }\end{array}$ & $\begin{array}{l}\text { Varía de tutor a } \\
\text { tutor - centrada } \\
\text { en los foros }\end{array}$ \\
\hline $\begin{array}{l}\text { Relación Estrategias de } \\
\text { aprendizaje disposicionales } \\
\text { y aprendizaje significativo }\end{array}$ & $\begin{array}{l}\text { Comunicación } \\
\text { por foros y } \\
\text { retroalimentación } \\
\text { de actividades }\end{array}$ & $\begin{array}{l}\text { Actividades que } \\
\text { promuevan } \\
\text { aprendizaje } \\
\text { significativo } \\
\text { Retroalimentación } \\
\text { del tutor }\end{array}$ & $\begin{array}{l}\text { Depende del } \\
\text { uso del } \\
\text { tutor- } \\
\text { herramientas } \\
\text { plataforma }\end{array}$ & $\begin{array}{l}\text { Posibilidad de } \\
\text { interacción (foros) } \\
\text { - claridad de la } \\
\text { información }\end{array}$ \\
\hline
\end{tabular}

Fuente: Elaboración propia

En esta triangulación de resultados se observa que existe una tendencia a promover aprendizajes significativos, con estrategias disposicionales que reconocen la interacción y el tipo de relación entre tutoraprendiz, siendo este un asunto diferente en cada docente. La retroalimentación de actividades, los foros de interacción, los chats y videos presentados en el aula virtual, resultan elementos destacados, que pueden ser objeto de mejora, o bien, pueden incluirse con otros elementos del aula virtual.

\section{Conclusiones}

Los hallazgos de este estudio son producto de la realidad estudiada y observada en un contexto educativo, realidad que se expone sin sesgos y con la cual, a partir de ella, se podría estimular la tarea docente en el aula para optar por nuevas directrices que sean efectivas al momento de impartir el conocimiento con estrategias. Aun cuando la diversificación de estrategias, métodos, técnicas, actividades son múltiples para ser desarrolladas se requiere de una planificación previa para ser aplicadas en los momentos donde hay que potenciar la habilidad y la motivación del estudiantado. Los hallazgos de este estudio no evidencian un proceso de diseño y planeación intencionada y normada de las estrategias disposicionales en todos los cursos analizados, pero sí una implementación que parte de los rasgos de espontaneidad e intuición del maestro sobre algunos de los contenidos y actividades instruccionales. Estos rasgos característicos y personales del y la docente debieran evidenciarse en una clase porque son una parte inherente y válida en la tarea formal del y la docente, sin embargo, la tarea del y la docente debe ser también organizada, sistematizada, diseñada, planificada, aplicada y evaluada, sin querer llegar a exceder el tradicionalismo acostumbrado de muchos docentes que no se dan oportunidad para usar sus habilidades espontaneas e intuitivas en el aula. 
La práctica educativa desde el diseño, implementación y evaluación de estrategias motivacionales dentro del entorno virtual de aprendizaje es importante en la relación tutor-aprendiz porque promueve el aprendizaje significativo, por ello, la identificación de las estrategias implementadas en estos tipos de estudios, podrían aportar al mejoramiento continuo de los procesos de planificación e implementación de las clases con estrategias y como consecuencia, contribuir a la motivación y a la formación integral de sus estudiantes.

En las declaraciones de cada participante de este estudio se pueden detectar que existen variantes en la práctica del proceso instruccional de cada docente del programa, esto sería un resultado obvio desde la perspectiva diferencial del conjunto docente, pero en medio de esas variantes individuales se detecta que existe la necesidad de un diseño, un plan, una implementación de formación y acompañamiento organizado siguiendo pautas y normas que rigen la autoridad de la educación y el seguimiento de las prácticas educativas en un aula virtual.

La planificación e implementación de estrategias disposicionales para promover el aprendizaje significativo resulta un asunto evidente para los distintos actores que interactúan en el aula virtual. Las mediaciones discursivas en la relación docente-estudiante, así como recursos gráficos y multimedia en los diseños instruccionales llevan a generar espacios de aprendizaje que conectan la motivación inicial del estudiantado con los objetivos o valores propuestos en las unidades temáticas por el y la docente, como ya se había señalado en estudios de Padilla, Rincón y Lagos (2016) y Pegalajar (2016). De este modo, la motivación del aprendiz y las estrategias de aprendizaje desarrolladas por cada docente se conjugan potenciando las posibilidades de apropiación del conocimiento a partir de su componente emotivo y experiencial.

Una de las principales barreras del entorno virtual es la comunicación asociada al componente motivacional en los diseños instruccionales analizados, las estrategias que apelan a la motivación se centran en los foros como medios de interacción de uso opcional (Valenzuela y Pérez, 2013). En ese sentido, la mediación discursiva del tutor en el proceso de enseñanza aprendizaje aprovecha el uso de la motivación por medio de la retroalimentación de actividades y cuestionamientos al inicio del curso, permitiendo que la motivación personal de cada aprendiz/a a la hora de elegir la carrera profesional se agilice (Meza et ál., 2016).

Los hallazgos de este estudio demostraron la existencia del (a) uso de estrategias disposicionales que han permitido el aprendizaje significativo de los contenidos y fue evidenciado durante las retroalimentaciones que brindó el personal docente en las comunicaciones sincrónicas y asincrónicas; (b) uso de un proceso intuitivo de parte del y la docente para aplicar sus estrategias; (c) uso de las estrategias motivacionales que cada docente emplea y que implican al mismo tiempo, el uso de contenidos textuales y gráficos, así como elementos evaluados por los entrevistados, como promotores de la motivación y aprendizajes significativos. Esto se detectó en el diseño de las actividades, las retroalimentaciones, así como en las herramientas sincrónicas y asincrónicas usadas en el entorno virtual. Herrera (2014), Meza et ál. (2016) y Valenzuela y Pérez (2013) destacan estos elementos como esenciales para el proceso de enseñanza-aprendizaje en el aula virtual, siendo promotores de otros elementos que facilitan el aprendizaje significativo, como son la motivación o valoración positiva del proceso, la autorregulación y el uso de herramientas tecnológicas de apoyo.

\section{ReFERENCIAS}

Aguilar, S. y Barroso, J. (2015). La triangulación de datos como estrategia en investigación educativa. Revista de Medios y Educación, 4(7), 73-88. doi: http://dx.doi.org/10.12795/pixelbit.2015.i47.05

Ahmed, Y., Ahmed, V., Miller, H.H., Gebremeskel, A. y Ebessa, D. (2018) Mapping inequality in access to meaningful learning in secondary education Ethiopia: implications for sustainable development. Journal Educational Studies, 45 (4). https://doi.org/10.1080/03055698.2018.1509777

Anaya, A. y Anaya, C. (2010). ¿Motivar para aprobar o para aprender? Estrategias de motivación del aprendizaje para las y los estudiantes. Tecnología, Ciencia y Educación, 25(1), 5-14. 
Andreú, J. (2001). Las técnicas de Análisis de Contenido: Una revisión actualizada. España: Centro de Estudios Andaluces. Recuperado de http://public.centrodeestudiosandaluces.es/pdfs/S200103.pdf

Angulo, P. (2005). Educación a distancia en el siglo XXI. Revista Apertura, 2(5), 60-75. Recuperado de http://www .redalyc.org/pdf/688/68800206.pdf

Arias, W.L y Oblitas, A. (2014). Aprendizaje por descubrimiento vs. Aprendizaje significativo: Un experimento en el curso de historia de la psicología. Boletín Académico Paulista de Psicología, 34(87), 455-471.

Ausubel, D., Novak, J.y Hanesian, H. (1983). Psicología educativa: Un punto de vista cognoscitivo. México D.F., México: Trillas.

Bardin, A. (2002). Análisis de Contenido. Madrid, España: Akal.

Boza, A. y Toscano, M. (2012). Motivos, actitudes y estrategias de aprendizaje: aprendizaje motivado en alumnos universitarios. Docente, Revista de currículum y formación del y la docente, 16(1), 126-139.

Cañedo, T. y Figueroa, I.E. (2013). La Práctica Docente en la Educación Superior: Una Mirada hacia su Complejidad. Sinéctica, 41, 1-18. Recuperado de http://bit.ly/2Xhi8rM

Carvalho, A. y De Oliveira, K.L. (2015). Students' Motivation for Learning in Virtual Learning Environments. Paidéia, 25(60), 105-113. doi: http://dx.doi.org/10.1590/1982-43272560201513

Díaz, F. y Hernández, G. (2002). Estrategias Docentes para un Aprendizaje Significativo. México D.F., México: Mc Graw Hill.

García, L. (2001). Bases conceptuales. En Educación a Distancia. De la Teoría a la Práctica. Madrid, España: Ariel Educación.

García B., Loredo J. y Carranza G. (2008). Análisis de la práctica educativa de las y los docentes: pensamiento, interacción y reflexión. Revista electrónica de investigación educativa (REDIE), 10, 1-15. Recuperado de http:/ /bit.ly/2VVNRgo

García, L. (2017). Educación a distancia y virtual: calidad, disrupción, aprendizajes adaptativo y móvil. Revista Iberoamericana de Educación a Distancia (RIED), 20(2), 9-25. Recuperado de http://revistas.uned.es/index.ph $\mathrm{p} /$ ried/article/view/18737.

Gargallo, B. (2000). Procedimientos, estrategias de aprendizaje, su naturaleza, enseñanza y evaluación. Valencia, España: Tirant lo Blanch.

Giroux, S. y Tremblay, G. (2009). Metodología de las Ciencias Humanas. México D.F., México: Fondo de Cultura Económica.

Hellberg, J. y Lindberg, S. (2017). Motivating students with authentic science experiences: changes in motivation for school science. Research in Science y Technological Education, 35(4), 409-42. doi: https://doi.org/10.1080/026 35143.2017.1322572

Hernández, R., Fernández, C. Baptista, P. (2010) Metodología de la Investigación. México D.F., México: McGraw-Hill Interamericana

Herrera, F. (2014). Motivación, estrategias de aprendizaje y rendimiento académico, en alumnos de Nutrición y Dietética de la Universidad del Desarrollo. Revista Educación, Ciencia y Salud, 11(1), $38 \# 46$.

Hwang, A. (2017). Online and hybrid learning. Journal of Management Education, 42(4). 557-563. Recuperado de h ttps://bit.ly/2EFV7qU

Íñiguez, L. (2006). Análisis del Discurso: Manual para las Ciencias Sociales. Barcelona, España: UOC.

Landa, P., Silva, A. y Guarnero, E. (2012). Factores Disposicionales en el Aprendizaje en la Psicología, una investigación apoyada por TIC. Revista electrónica de Psicología Iztacala, 15(4), 1476-1493. Recuperado de http://www.revi stas.unam.mx/index.php/repi

Maor, D. (2003). Teacher's and students' perspectives on on-line learning in a social constructivist learning environment. Pedagogy and Education, 12(2). 201-218. doi:https://doi.org/10.1080/14759390300200154

Marín, V. y Maldonado, G. (2011). El alumnado universitario cordobés y la plataforma virtual Moodle. Pixel-Bit. Revista de Medios y Educación, 38, 121-128. Recuperado de http://www.redalyc.org/articulo.oa?id=36816200 009 
Marcela Duarte- Herrera, et al. Estrategias disposicionales y aprendizajes Significativos en el aU...

Martins, M. y Unger, L.M. (2017). Virtual Teaching Dispositions in an Open Distance Learning Environment: Origins, Measurement and Impact. Africa Education Review, (14)2, 156-173. doi: https://doi.or/10.1080/181 46627.2017.1294971

Meza, L., Torres, S. y Lara, J. (2016). Estrategias de aprendizaje emergentes en la modalidad e-learning. Revista de Educación a Distancia, 48(5), 1-21. Recuperado de http://www.um.es/ead/red/48/meza.pdf.

Mieles, M., Tonon, G. y Alvarado S. (2012). Investigación cualitativa: el análisis temático para el tratamiento de la información desde el enfoque de la fenomenología social. Universitas Humanistica, 74, 195-225. Recuperado de http://www.redalyc.org/articulo.oa?id=79125420009

Monereo, C. (2000). Estrategias de Aprendizaje. Madrid, España: Aprendizaje Visor.

Moreira, M.A. (2000). Aprendizaje Significativo: Teoria y Práctica. Madrid, España: Aprendizaje Visor

Okuda, M. y Gómez, C. (2005). Métodos en investigación cualitativa: triangulación. Revista Colombiana de Psiquiatría, 14(1), 118-124. Recuperado de http://www.redalyc.org/pdf/806/80628403009.pdf

Padilla, J., Rincón, D. y Lagos, J. (2016). La poiesis en la facilitación del aprendizaje para el uso de las TIC en Educación Superior. Revista Academia \& Virtualidad 9(2), 138-155. doi: https://doi.org/10.18359/ravi.1760

Pegalajar, M.C. (2016). Estrategias de aprendizaje en alumnado universitario para la formación presencial y semipresencial. Revista Latinoamericana de Ciencias Sociales, Niñez y Juventud, 14 (1), 659-676. Recuperado de https://dialnet.unirioja.es/servlet/articulo?codigo $=5382049$

Tintaya, P. (2010). Estrategias orientadoras del aprendizaje. Revista de Investigación Psicológica, 6, 5-102. Recuperado de http://bit.ly/2XcryVs

Valenzuela, B. y Pérez, M.V. (2013). Aprendizaje autorregulado a través de la plataforma virtual Moodle. Educación y educadores, 16(1), 66-79.

Vivas, N. (2010). Estrategias de aprendizaje. Gondola. Enseñanza y Aprendizaje de las Ciencias, 1(5), 27-37. Recuperado de https://bit.ly/2HK57l3

CC BY-NC-ND 